\title{
A experiência brasileira na construção de Índices de Qualidade de Vida Urbana: planejamento e gestão urbana
}

The Brazilian experience in the construction of Urban Quality of Life Indexes: urban planning and management

La experiencia brasileña en la construcción de Índices de Calidad de Vida Urbana: planificación y gestión urbana

Fabio Silva Santos

Historiador, Mestre em Relações Internacionais, Universite D'Aux-Marseille-I, Brasil cidades.vivas@gmail.com

Douglas Gallo

Professor Mestre, IFSP, Brasil Doutorando em Urbanismo, PROURB/FAU/UFRJ, Brasil douglas.luciano@yahoo.com.br 


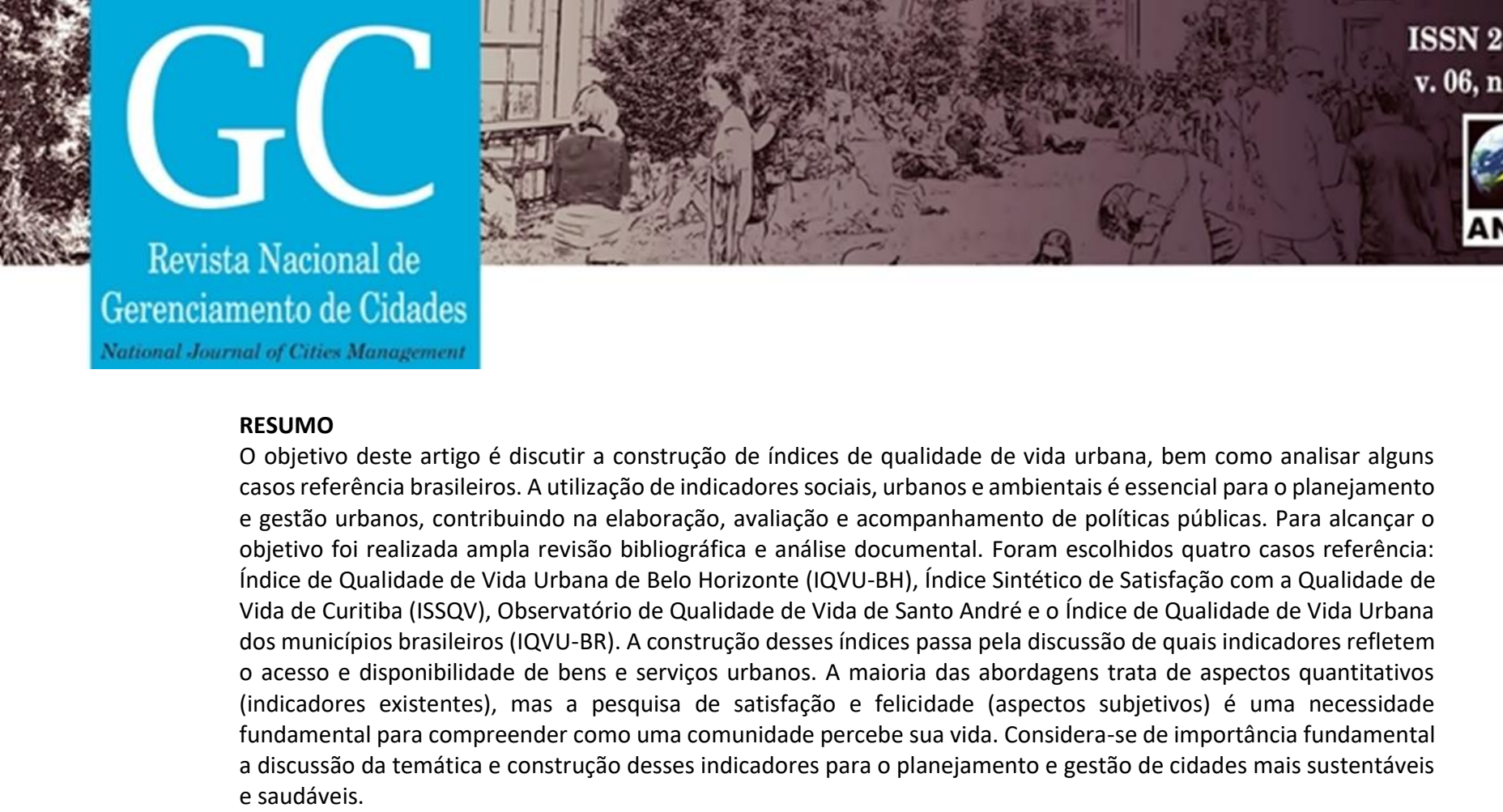

PALAVRAS-CHAVE: Qualidade de vida urbana. Indicadores sociais. Planejamento urbano.

\begin{abstract}
This study aimed to discuss the construction of Urban Quality of Life Indexes, as well to analyze some Brazilian reference cases. The use of social, urban and environmental indicators is essential for urban planning and management, contributing to the elaboration, evaluation and monitoring of public policies. A bibliographic review and documentary analyses were carried out to respond to this objective. Four reference cases were chosen: the Belo Horizonte Urban Quality of Life Index (IQVU-BH), the Synthetic Index of Satisfaction with Quality of Life of Curitiba (ISSQV), the Santo André Quality of Life Observatory and the Brazilian Urban Quality of Life Index (IQVU-BR). The construction of these indexes involves the discussion of which indicators reflect the access and availability of urban goods and services. Most approaches deal with quantitative aspects (secondary indicators), but the search for satisfaction and happiness (subjective aspects) is a fundamental need to understand how a community perceives its life. It is considered of fundamental importance to discuss the thematic and construction of these indicators for the planning and management of more sustainable and healthy cities.
\end{abstract}

KEYWORDS: Urban quality of life. Social indicators. Urban planning.

\title{
RESUMEN
}

El propósito de este ensayo es discutir la construcción de índices de calidad de vida urbana, así como analizar algunos casos referencia brasileños. La utilización de indicadores sociales, urbanos y ambientales es esencial para la planificación y gestión urbanas, contribuyendo en la elaboración, evaluación y seguimiento de políticas públicas. Para alcanzar el objetivo se realizó amplia revisión bibliográfica y análisis documental. Se eligieron cuatro casos de referencia: Índice de Calidad de Vida Urbana de Belo Horizonte (IQVU-BH), Índice Sintético de Satisfacción con la Calidad de Vida de Curitiba (ISSQV), Observatorio de Calidad de Vida de Santo André y el Índice de Calidad de Vida Urbana de los municipios brasileños (IQVU-BR). La construcción de estos índices pasa por la discusión de cuáles indicadores reflejan el acceso y disponibilidad de bienes y servicios urbanos. La mayoría de los enfoques trata de aspectos cuantitativos (indicadores existentes), pero la investigación de satisfacción y felicidad (aspectos subjetivos) es una necesidad fundamental para comprender cómo una comunidad percibe su vida. Se considera de importancia fundamental la discusión de la temática y construcción de esos indicadores para la planificación y gestión de ciudades más sostenibles y saludables.

PALABRAS CLAVE: Calidad de vida urbana. Indicadores sociales. Planificación urbana. 

urbanos, seu alcance e cobertura, bem como as necessidades humanas objetivas. Pressupõe-se também considerar os usos e apropriações dos espaços públicos e a sociabilidade, relacionadas às necessidades subjetivas e de percepção dos indivíduos integrados ou não à sociedade, bem como os vínculos, contatos, sentimento de pertencimento e participação na vida coletiva (VITTE e KEINERT, 2009).

Existem novos desafios para a gestão local e para as políticas públicas no intuito de resgatar o papel da cidade como lugar eletivo do processo civilizador. Neste aspecto, a dimensão política, relacionando aspectos do planejamento urbano e da gestão pública deve buscar o fortalecimento da cidadania e dos direitos fundamentais das pessoas e dos direitos sociais. $\mathrm{Na}$ atualidade, o ambiente urbano, como espaço síntese da civilização, se torna um espaço privilegiado para experimentações e sistematização de ideais e informações que reflitam o dinamismo das transformações quotidianas nas cidades. A complexidade e diversidade da vida, nas diversas escalas, do local ao global, misturam tempos espaciais diferentes e articulados, que se redefinem em espaços locais surpreendentes (MONTE-MÓR, 2015).

Desta forma, ao refletir sobre a qualidade de vida como parâmetro para avaliação de políticas públicas, do planejamento urbano e da gestão pública, particularmente a municipal, é necessário observarmos as formas de avaliá-la. Particularmente em países com imensas desigualdades e grandes iniquidades sociais como o Brasil, onde a construção de indicadores de qualidade de vida urbana tem importância fundamental.

O objetivo deste artigo é discutir a construção de indicadores para avaliação da qualidade de vida urbana, bem como analisar alguns casos referência que propuseram índices locais ou regionais como instrumentos de planejamento e gestão. Para atingir o objetivo foi realizada ampla revisão bibliográfica e análise de casos referência, na realidade brasileira.

\section{QUALIDADE DE VIDA URBANA}

O conceito de "qualidade de vida urbana" está associado ao de "qualidade de vida", referente ao bem-estar das pessoas e à sua satisfação com as circunstâncias de vida. O qualificador "urbana" remete às cidades e aos problemas do seu processo de desenvolvimento (NAHAS, 2015). Essa questão, seja qual for a comunidade a que se refere, das cidades aos vastos bolsões de pobreza que encerram, é complexa e multifacetada. Compreende tanto a distribuição dos bens de cidadania (bens e direitos que uma sociedade, num determinado tempo, julga essenciais), quanto bens coletivos de natureza menos palpável, mas com repercussão sobre o bem-estar social (SOUZA, 1982).

A preocupação com a qualidade de vida urbana assumiu importância no debate político e científico após a década de 1960, devido ao grande crescimento das cidades. O modelo de 
desenvolvimento adotado aumentou a desigualdade na distribuição de bens e serviços, com forte impacto nas condições de vida da população e no meio ambiente. Desenvolveu-se a noção de sustentabilidade do desenvolvimento humano, trazendo novo significado para o conceito de qualidade de vida (NAHAS, 2015).

A temática vem ganhando espaço nas discussões sobre os objetivos a serem alcançados ou preservados pelas políticas públicas. Uma perspectiva muito importante é da possibilidade de sua utilização no planejamento econômico, social e urbano. O conceito de qualidade de vida e seus indicadores podem servir ao planejamento atuando como parâmetro do grau de cobertura das necessidades individuais ou de grupos sociais, detectando desigualdades e embasando a elaboração de estratégias para melhorar o bem-estar (VITTE et al, 2002).

A noção de qualidade de vida é construída a partir da percepção que cada comunidade tem sobre viver com qualidade. É um conceito complexo, de caráter subjetivo e qualitativo, exprimindo juízos de valor, tendo, pois, uma natureza política e ética (KEINERT, KARRUZ e KARRUZ, 2002). A ideia introduz uma valorização de horizontes desejáveis por determinados grupos sociais, com diferentes níveis de exigência e aspirações, mais afeitos à percepção dos indivíduos. Desta forma, o conceito de qualidade de vida guarda relação com a satisfação das necessidades humanas e com a capacidade que uma comunidade tenha de desfrutar uma vida média longa e saudável (VITTE, 2009).

A maioria das abordagens ao tema consideram o atendimento das necessidades básicas, ou parâmetros objetivos, a percepção da população, ou parâmetros subjetivos, e os valores vinculados ao desenvolvimento sustentável (KEINERT, KARRUZ e KARRUZ, 2002). Allardt (1998), propõe um enfoque diferente, baseado em condições necessárias ao desenvolvimento humano, representadas pelos verbos 'Ter', 'Amar' e 'Ser'. Estas são palavras de efeito para chamar a atenção sobre condições tanto materiais como não materiais de um sistema de indicadores de avaliação da qualidade de vida e bem-estar de uma sociedade.

O verbo 'Ter' relaciona-se às condições materiais necessárias à sobrevivência e para evitar a miséria, cobrindo necessidades como nutrição, ar, água, proteção contra o clima, o ambiente e enfermidades. O verbo 'Amar' refere-se à necessidade de relacionar-se com outras pessoas e formar identidades sociais. Já o verbo 'Ser' está ligado à necessidade de integrar-se à sociedade e viver em harmonia com a natureza (ALLARDT, 1998).

\section{A CONSTRUÇÃO DE INDICADORES SOCIAIS}

A qualidade de vida pode ser melhorada mesmo com baixos níveis de renda mediante adequados programas de serviços sociais. Serviços de saúde e educação de qualidade, conduzidos pelo custeio público podem impactar na vida da população mesmo sem esperar que 
esta "fique rica" primeiro, evidenciando a importância de políticas públicas e seu monitoramento mediando indicadores sociais, voltadas para a qualidade de vida (SEN, 2010).

O termo indiciadores sociais surgiu na década de 1960, nos Estados Unidos, como componentes do sistema social que descrevem a si próprios. Seu aparecimento e desenvolvimento está intrinsecamente ligado ao planejamento do setor público (JANNUZZI, 2002a). Para as políticas públicas, são medidas que possibilitam a operacionalização de conceitos abstratos ou demandas de interesse programáticas. Os indicadores têm papel fundamental no desenho, implementação e avaliação das políticas públicas (PARAHOS et al, 2013).

Importante lembrar que os indicadores apenas indicam, não substituindo o conceito social subjacente (JANNUZZI, 2002a; 2002b). Para que seja útil à formulação de políticas públicas é necessário que expressem a capacidade do município em oferecer qualidade de vida aos seus moradores, avaliando serviços e equipamentos localizados geograficamente. Para Nahas (2015: 28):

Torna-se indispensável a construção de indicadores georreferenciados no espaço intraurbano, permitindo também ao gestor público local a identificação de prioridades espaciais para certos investimentos, especialmente localização de equipamentos de forma a promover um acesso mais equitativos a serviços e bens, promovendo a inclusão social e a equidade.

De acordo com Cary e Jany-Catrice (2015), os indicadores sociais devem expressar conceitos objetivos complexos como a sustentabilidade social, desenvolvimento sustentável, qualidade de vida e bem-estar.

Existem duas principais abordagens na elaboração de indicadores de qualidade de vida urbana, uma objetiva e outra subjetiva. Na abordagem subjetiva considera-se que a qualidade de vida seja determinada pela percepção que o indivíduo tem de sua própria vida, e para tanto deveria ser aferida apenas por indicadores subjetivos, sendo os principais indicadores as medidas de satisfação ou felicidade. A abordagem objetiva considera que as condições objetivas de vida, ou seja, os recursos aos quais os indivíduos têm acesso, tais como renda, propriedade, conhecimento, relações sociais e segurança, determinam a qualidade de vida. Os indicadores objetivos avaliam características do ambiente urbano, como equipamentos, serviços e redes de infraestrutura, utilizando-se de dados secundários (NAHAS, 2015).

Quando se diferencia entre quantidade e qualidade de vida, ambas gradações são importantes para determinar quão boa é a vida das pessoas. Indivíduos cujas vidas tem grande qualidade, seja qual medida utilizada, se tiverem uma morte prematura não terão uma vida muito boa. Nossos planos de vida são desenvolvidos o mais tardar na adolescência e comumente são redefinidos conforme a vida se desenvolve. Ao terminar a vida prematuramente, devido a 
enfermidades ou padecimentos, a pessoa não só perde as experiências, felicidade e satisfação que teria nos anos vindouros, mas também perde a oportunidade de completar projetos de longo prazo e de alcançar a vida em toda sua conformação, coerência e conclusão de seu planejamento. Assim, completar e terminar um plano de vida é o que ajuda as pessoas idosas, próximas da morte, a sentir que a viveram uma vida plena e completa (BROCK, 1998).

Um sistema de indicadores que considere aspectos conceituais e metodológicos adequados podem ser úteis para o planejamento municipal, subsidiando a formulação de políticas públicas e monitorando a qualidade de vida, especialmente em grandes cidades. Para tanto, o maior desafio que as administrações municipais enfrentam é a manutenção de sistemas de informação que possam disponibilizar indicadores fidedignos e de forma contínua, a fim de balizar e monitorar o desenvolvimento de um índice de qualidade de vida urbana (NAHAS, 2009).

\section{3. ÍNDICES DE QUALIDADE DE VIDA URBANA}

Segundo Nahas et al (2006), a mensuração da qualidade de vida urbana, na contemporaneidade, deve incluir três elementos fundamentais:

i. Dimensionamento da equidade no acesso social e espacial da população aos bens e serviços urbanos.

ii. Avaliação da qualidade ambiental, partindo de aspectos socioambientais e aspectos ambientais "stricto senso".

iii. Produção de elementos para discussão da sustentabilidade no desenvolvimento humano.

\section{1 Índice de Qualidade de Vida Urbana (IQVU), Belo Horizonte/MG}

O IQVU foi desenvolvido pela prefeitura de Belo Horizonte/MG, juntamente com a Pontifícia Universidade Católica de Minas Gerais (PUC MG), na década de 1990. É uma medida de acesso espacial a recursos urbanos, gerando um índice intra-urbano composto por indicadores georreferenciados em Unidades de Planejamento (UP) da cidade (NAHAS e ESTEVES, 2015).

O índice monitora multidimensionalmente e quantifica as desigualdades espaciais de disponibilidade de bens e serviços no tecido urbano, avaliando o nível de infraestrutura e delimitando áreas prioritárias para investimentos públicos. Seu primeiro cálculo foi publicado em 1996, gerando uma série histórica. Em 2006 teve sua composição temática alterada, sendo calculado para 79 unidades de planejamento, composto por dez variáveis divididas em 33 indicadores (Quadro 1). Ao final é gerado um valor do índice, variando de 0 a 1 , sendo 1 o valor "ideal", significando pleno acesso aos bens e serviços, o que corresponderia a uma excelente 
qualidade de vida urbana (GUERRA, 2002; NAHAS, 2009; BELO HORIZONTE, 2015; NAHAS e ESTEVES, 2017).

Quadro 1: Relação dos Indicadores que compõe o IQVU de Belo Horizonte/MG

\begin{tabular}{|c|c|c|}
\hline VARIÁVEL & COMPONENTE & INDICADOR \\
\hline \multirow{2}{*}{ 1. ABASTECIMENTO } & \multirow{2}{*}{$\begin{array}{l}1.1 \text { Equipamentos de } \\
\text { abastecimento }\end{array}$} & 1.1.1 Hiper e Supermercados \\
\hline & & 1.1.2. Mercearias e similares \\
\hline \multirow{4}{*}{ 2. CULTURA } & \multirow{4}{*}{$\begin{array}{l}\text { 2.1 Comércio e Serviços } \\
\text { Culturais }\end{array}$} & 2.1.1 Distribuição de equipamentos \\
\hline & & 2.1.2 Livrarias e papelarias \\
\hline & & 2.1.3 Locadores por $1000 \mathrm{hab}$ \\
\hline & & 2.1.4 Bancas de revistas \\
\hline \multirow{5}{*}{ 3. EDUCAÇÃO } & 3.1 Educação Infantil & $\begin{array}{l}\text { 3.1.1 Percentual de alunos matriculados na Educação } \\
\text { Infantil }\end{array}$ \\
\hline & \multirow[t]{2}{*}{ 3.2 Ensino Fundamental } & $\begin{array}{l}\text { 3.2.1 Percentual de alunos matriculados no Ensino } \\
\text { Fundamental }\end{array}$ \\
\hline & & 3.2.2 Índice de Aproveitamento no Ensino Fundamental \\
\hline & \multirow[t]{2}{*}{ 3.3 Ensino Médio } & $\begin{array}{l}\text { 3.3.1 Percentual de alunos matriculados no Ensino } \\
\text { Médio }\end{array}$ \\
\hline & & 3.3.2 Índice de Aproveitamento no Ensino Médio \\
\hline 4. ESPORTES & $\begin{array}{l}\text { 4.1 Espaços públicos para } \\
\text { recreação }\end{array}$ & $\begin{array}{l}\text { 4.1.2 Quadras, campos, academias a céu aberto, } \\
\text { academias da cidade e outros equipamentos por } 1000 \\
\text { hab }\end{array}$ \\
\hline \multirow{3}{*}{ 5. HABITAÇÃO } & \multirow{2}{*}{ 5.1 Qualidade da Habitação } & 5.1.1 Área residencial adequada por habitante \\
\hline & & 5.1.2 Padrão de Acabamento \\
\hline & 5.2 Segurança Habitacional & 5.2.1 Índice do Risco Geológico do Terreno \\
\hline \multirow{5}{*}{$\begin{array}{l}6 . \\
\text { INFRAESTRUTURA } \\
\text { URBANA }\end{array}$} & 6.1 Salubridade Ambiental & 6.1.1 Índice de Salubridade Ambiental \\
\hline & 6.2 Energia Elétrica & 6.2.1 Fornecimento de energia elétrica \\
\hline & 6.3 Pavimentação & 6.3.1 Possibilidade de acesso \\
\hline & \multirow{2}{*}{ 6.4 Transporte Coletivo } & 6.4.1 Número de veículos por 1000 hab \\
\hline & & 6.4.2 Intervalo das linhas por UP \\
\hline \multirow{3}{*}{ 7. MEIO AMBIENTE } & 7.1 Conforto Acústico & 7.1.1 Tranquilidade sonora \\
\hline & 7.2 Qualidade do ar & 7.2.1 Ausência de coletivos poluidores \\
\hline & 7.3 Área verde & 7.3.1 Área verde por habitante \\
\hline \multirow{4}{*}{ 8. SAÚDE } & \multirow{3}{*}{8.1 Atenção à Saúde } & 8.1.1 Centros de Saúde por 1000 hab \\
\hline & & 8.1.2 Outros Equipamentos de Assistência Médica \\
\hline & & 8.1.3 Equipamentos Odontológicos \\
\hline & 8.2 Vigilância à Saúde & 8.2.1 Ausência de Anos Potenciais de Vida Perdidos \\
\hline \multirow{6}{*}{$\begin{array}{l}\text { 9. SERVIÇOS } \\
\text { URBANOS }\end{array}$} & \multirow{3}{*}{ 9.1 Serviços Pessoais } & 9.1.1 Agências Bancárias \\
\hline & & 9.1.2 Postos de Combustível \\
\hline & & 9.1.3 Farmácias \\
\hline & \multirow{3}{*}{$\begin{array}{l}\text { 9.2 Serviços de Comunicação } \\
\text { e Tecnologia }\end{array}$} & 9.2.1 Correios \\
\hline & & 9.2.2 Espaços públicos para inclusão digital \\
\hline & & 9.2.3 Telefones públicos \\
\hline \multirow{3}{*}{$\begin{array}{l}\text { 10. SEGURANÇA } \\
\text { URBANA }\end{array}$} & 10.1 Segurança Pessoal & 10.1.1 Ausência de crimes contra a pessoa \\
\hline & 10.2 Segurança Patrimonial & 10.2.1 Ausência de crimes contra o patrimônio \\
\hline & 10.3 Segurança no Trânsito & 10.3.1 Ausência de acidentes de trânsito \\
\hline
\end{tabular}

Fonte: BELO HORIZONTE, 2015

\section{2 Índice Sintético de Satisfação da Qualidade de Vida (ISSQV), Curitiba/PR}


Em 1987 foi criado o Índice Sintético de Satisfação da Qualidade de Vida (ISSQV), em Curitiba, elaborado pelo Instituto de Pesquisa e Planejamento Urbano de Curitiba (Ippuc). É um indicador composto e georreferenciado nos 75 bairros da cidade, visando expressar o acesso da população à: habitação, saúde, educação e transporte. A elaboração deste índice estabeleceu hierarquias para os bairros, indicando aqueles com melhor acesso às dimensões analisadas e também os mais carentes, possibilitando a identificação e mensuração espacial das necessidades urbanas (NAHAS, 2009).

Com a inserção da cidade na Rede Mundial de Municípios Saudáveis, a partir de 1996 intensificou-se as discussões sobre a qualidade de vida em Curitiba. Buscando contemplar o maior número de áreas do contexto urbano e suas potencialidades, os indicadores de impacto na vida da população e no ambiente urbano (Quadro 2) são monitorados para: caracterizar os diferenciais intra-urbanos de qualidade de vida; consolidar sua monitoração constante; detectar áreas que necessitem de maior intervenção; construir mapas temáticos; subsidiar o planejamento urbano e criação de políticas públicas específicas; efetivar um pensamento que busque a equidade no espaço urbano; e disseminar informações para ampliar o conhecimento coletivo (RIBEIRO e MELLO, 2002).

Quadro 2: Variáveis e indicadores de análise da Qualidade de Vida em Curitiba/PR

\begin{tabular}{|c|c|}
\hline VARIÁVEL & INDICADOR \\
\hline \multirow{3}{*}{ 1. HABITAÇÃO } & 1.1 Domicílios em aglomerados subnormais \\
\hline & 1.2 Pessoas por domicílio \\
\hline & 1.3 Domicílios com coleta de lixo \\
\hline \multirow{7}{*}{ 2. SAÚDE } & 2.1 Mortalidade infantil \\
\hline & 2.2 Baixo peso ao nascer \\
\hline & 2.3 Mortalidade geral padronizada \\
\hline & 2.4 Esperança de vida ao nascer \\
\hline & 2.5 Incidência de tuberculose \\
\hline & 2.6 Incidência de AIDS \\
\hline & 2.7 Mortalidade $<$ de 5 anos \\
\hline \multirow{4}{*}{ 3. EDUCAÇÃO } & 3.1 Taxa de reprovação \\
\hline & 3.2 Taxa de abandono \\
\hline & 3.3 Taxa de sucesso \\
\hline & 3.4 Distorção idade/série \\
\hline \multirow{3}{*}{ 4. TRANSPORTE } & 4.1 Frequência \\
\hline & 4.2 Acesso a rede integrada de transporte \\
\hline & 4.3 Cumprimento das viagens \\
\hline \multirow{4}{*}{$\begin{array}{l}\text { 5. AGENTE DA VARIABILIDADE } \\
\text { DOS BENEFÍCIOS SOCIAS }\end{array}$} & 5.1 Renda Média \\
\hline & 5.2 Área Típica de Variação \\
\hline & 5.3 Concentração (Índice) \\
\hline & 5.4 Concentração (Distribuição do Fundo Geral de Renda \\
\hline
\end{tabular}

Fonte: RIBEIRO e MELLO, 2002 


\subsection{Observatório da Qualidade de Vida de Santo André, Santo André/SP}

A prefeitura de Santo André conta com longa experiência no levantamento de informações, possuindo um departamento de estatística desde 1945. O acesso aos bancos de dados socioeconômicos e ambientais possibilitaram a construção do Observatório da Qualidade de Vida, consolidando dados e operacionalizando um sistema de formulação, gestão e avaliação de políticas públicas (PRADO e KEINERT, 2009).

O conceito de um observatório desenvolve-se como uma metodologia de monitoramento, com capacidade de analisar indicadores, confrontando-os para articular informações e oferecer à comunidade um retrato interpretado para que os diferentes agentes econômicos, políticos, sociais e culturais possam posicionar-se a respeito. Sua principal função é realizar diagnóstico, não apenas criar mapas, buscando consolidar indicadores concretos e empíricos capazes de traduzir na prática os conceitos teóricos envolvidos (KARRUZ, KEINERT e KEINERT, 2002; KARRUZ e KEINERT, 2009).

Na operacionalização do conceito de qualidade de vida partiu-se da noção de longevidade, considerando a capacidade de uma comunidade desfrutar de uma vida média longa de forma saudável. Desta forma, a qualidade de vida seria expressa numa relação entre os anos vividos e o bem-estar, entendido como um conjunto de elementos da vida social e urbana que favorecem ou dificultam a experiência de uma vida saudável, incluindo aspectos urbanísticos, de cidadania, saúde pública, educação, segurança, entre outros (KARRUZ e KEINERT, 2009).

Juntamente com os dados objetivos para a construção do Índice de Qualidade de Vida, foram considerados aspectos subjetivos, relacionados à percepção que os moradores do município tinham de determinados fatores e aspectos relacionados à felicidade e qualidade de vida (Quadro 3). Felicidade entendida como o que é importante na vida das pessoas. 
mensuração da acessibilidade (Tabela 1). Com isso, foram gerados 26 temas que serviram de referência para estabelecer um modelo de cálculo do índice e proposição dos indicadores (Tabela 2).

Tabela 1: Aspectos teórico-metodológicos a serem considerados na construção do índice, levantados na Consulta Nacional

\begin{tabular}{lc}
\hline Quanto à oferta de serviços urbanos & Sim (\%) \\
\hline Que o índice reflita a diversidade do conjunto dos serviços ofertados & 100,0 \\
Os indicadores avaliem também a regularidade da oferta & 95,6 \\
Que se considere o fator de sustentabilidade como o de maior relevância & 91,1 \\
Que se considere a inclusão de medidas subjetivas de satisfação com a oferta e/ou com & 82,2 \\
o acesso & \\
\hline Quanto a questões territoriais & Sim (\%) \\
\hline Que se considere o porte das cidades no cálculo do índice & 97,8 \\
Que se considere a inserção regional, ou seja, a articulação do município e sua função na & 95,6 \\
rede urbana & 95,6 \\
Que se considere as diferenças e peculiaridades regionais na delimitação da abrangência & \\
dos temas e na formulação dos indicadores & Sim (\%) \\
\hline Quanto à acessibilidade a serviços, considerar também os aspectos demográficos & 95,7 \\
\hline Faixa etária & 78,0 \\
Gênero & 70,0 \\
\hline Raça/cor & Sim (\%) \\
\hline Quanto ao enfoque teórico dos indicadores & 81,8 \\
\hline Formular os indicadores a partir da abordagem dos Direitos Humanos
\end{tabular}




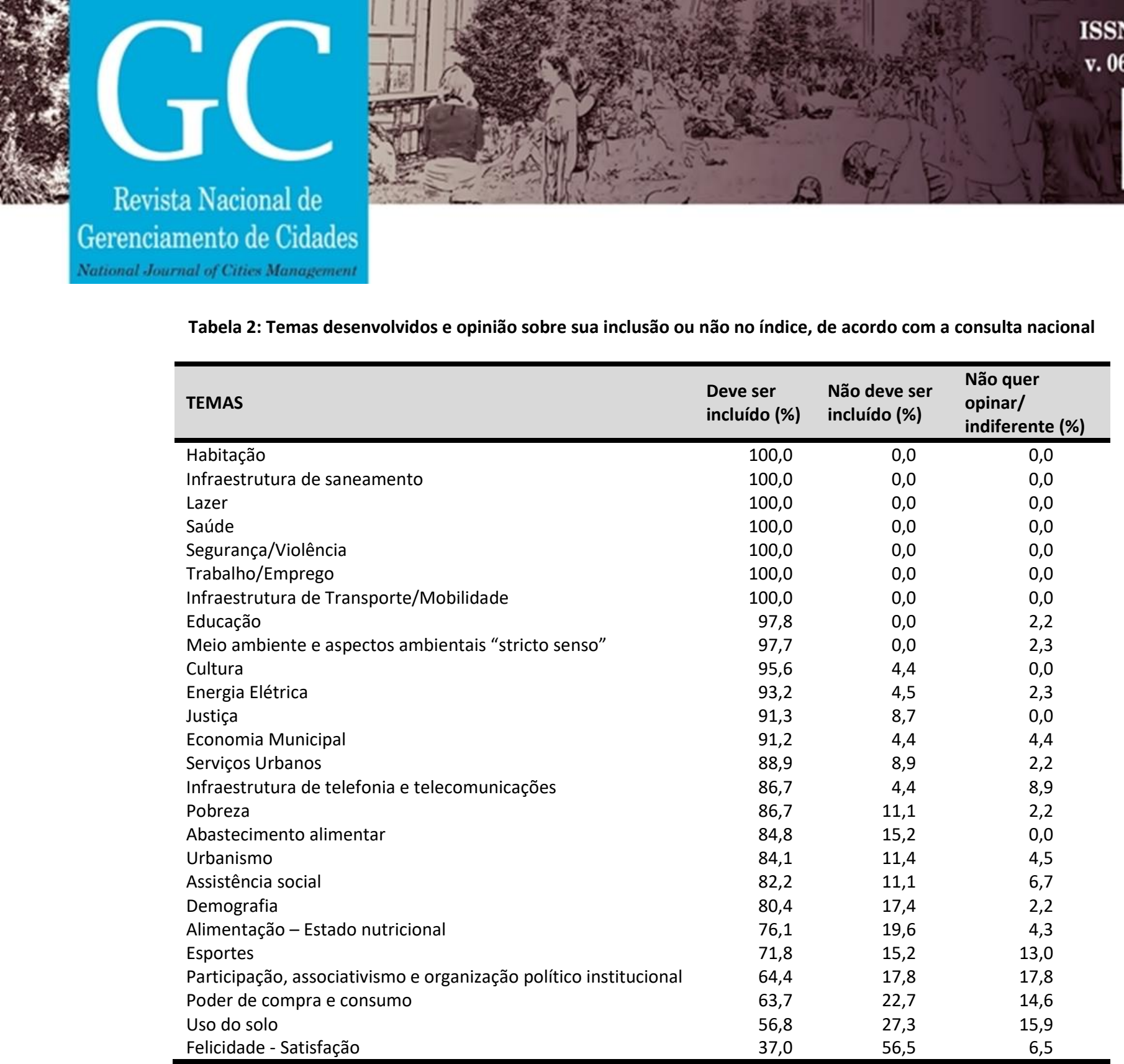

Felicidade - Satisfação

Fonte: NAHAS et al, 2006

Ao final do processo foram propostos um total de 431 indicadores ideais para a composição do índice (Tabela 3), sendo classificados entre indicadores de quantidade ou de qualidade da oferta de serviços urbanos. 
garantir cidades mais sustentáveis e saudáveis. Acreditamos que os casos referência analisados neste estudo contribuem para a discussão e valorização da abordagem da qualidade de vida urbana para o planejamento e gestão urbanos, por meio de políticas públicas mais integrais e intersetoriais.

\section{REFERÊNCIAS BIBLIOGRÁFICAS}

ALLARDT, Erik. Tener, amar, ser: una alternativa al modelo sueco de investigación sobre el Bienestar. In: NUSSBAUM, Martha C.; SEN, Amartya. La calidad de vida. México: Fondo de Cultura Económica, 1998.

BELO HORIZONTE, Prefeitura Municipal. Relatório geral sobre o cálculo do Índice de Qualidade de Vida Urbana de Belo Horizonte para 2014. Belo Horizonte: Secretaria Municipal de Planejamento, Orçamento e Gestão, 2015.

BROCK, Dan. Medidas de la calidad de vida en el cuidado de la salud y la ética médica. In: NUSSBAUM, Martha C.; SEN, Amartya. La calidad de vida. México: Fondo de Cultura Económica, 1998.

CARY, Paul; JANY-CATRICE, Florence. Nouveaux indicateurs de richesse, nouveaux imaginaires politiques? In: NAHAS, Maria Inês Pedrosa. Qualidade de vida urbana: abordagens, indicadores e experiências internacionais. Belo Horizonte: C/Arte, 2015.

FARAH, Marta Ferreira Santos. Inovação e governo local no Brasil contemporâneo. In: JACOBI, Pedro; PINHO, José Antonio. Inovação no campo da gestão pública local: novos desafios, novos patamares. Rio de Janeiro: Editora FGV, 2006.

GUERRA, Leonardo Pontes. A experiência de Belo Horizonte com planejamento, gestão e análise da cidade através do Sistema Local de Informações. In: KEINERT, Tânia Margarete Mezzomo; KARRUZ, Ana Paula. Qualidade de vida: observatórios, experiências e metodologias. São Paulo: Annablume/FAPESP, 2002.

JANNUZZI, Paulo de Martino. Considerações sobre o uso, mau uso e abuso dos indicadores sociais na formulação e avaliação de políticas públicas municipais. RAP. 36(1): 51-72, jan./fev. 2002.

JANUZZI, Paulo de Martino. Repensando a prática de uso de indicadores sociais na formulação e avaliação de políticas públicas municipais. In: KEINERT, Tânia Margarete Mezzomo; KARRUZ, Ana Paula. Qualidade de vida: observatórios, experiências e metodologias. São Paulo: Annablume/FAPESP, 2002b.

KARRUZ, Ana Paula; KEINERT, Tânia Margarete Mezzomo. Do abstrato ao concreto: a proposta do Observatório da Qualidade de Vida de Santo André para operacionalização da noção de qualidade de vida, via estudo especializado da longevidade. In: VITTE, Claudete de Castro Silva; KEINERT, Tânia Margarete Mezzomo. Qualidade de vida, planejamento e gestão urbana: discussões teórico-metodológicas. Rio de Janeiro: Bertrand Brasil, 2009.

KARRUZ, Ana Paula; KEINERT, Ruben Cesar; KEINERT, Tânia Margarete Mezzomo. O processo de construção do Observatório da Qualidade de Vida de Santo André: identificação e superação de desafios. In: KEINERT, Tânia Margarete Mezzomo; KARRUZ, Ana Paula. Qualidade de vida: observatórios, experiências e metodologias. São Paulo: Annablume/FAPESP, 2002.

KEINERT, Tânia Margarete Mezzomo; KARRUZ, Ana Pauloa; KARRUZ, Silvia Maria. Sistemas locais de informação e a gestão pública da qualidade de vida nas cidades. In: KEINERT, Tânia Margarete Mezzomo; KARRUZ, Ana Paula. Qualidade de vida: observatórios, experiências e metodologias. São Paulo: Annablume/FAPESP, 2002.

MONTE-MÓR, Roberto Luís. Prefácio. In: NAHAS, Maria Inês Pedrosa. Qualidade de vida urbana: abordagens, indicadores e experiências internacionais. Belo Horizonte: C/Arte, 2015. 
NAHAS, Maria Inês Pedrosa. Indicadores de qualidade de vida urbana: aspectos teórico-metodológicos. In: NAHAS, Maria Inês Pedrosa. Qualidade de vida urbana: abordagens, indicadores e experiências internacionais. Belo Horizonte: C/Arte, 2015

NAHAS, Maria Inês Pedrosa; ESTEVES, Otávio de Avelar. Metodologia do índice de qualidade de vida urbana e suas aplicações. In: NAHAS, Maria Inês Pedrosa. Qualidade de vida urbana: abordagens, indicadores e experiências internacionais. Belo Horizonte: C/Arte, 2015.

NAHAS, Maria Inês Pedrosa. Indicadores intra-urbanos como instrumentos de gestão da qualidade de vida urbana em grandes cidades: uma discussão teórico-metodológica. In: VITTE, Claudete de Castro Silva; KEINERT, Tânia Margarete Mezzomo. Qualidade de vida, planejamento e gestão urbana: discussões teórico-metodológicas. Rio de Janeiro: Bertrand Brasil, 2009.

NAHAS, Maria Inês Pedrosa; et al. Metodologia de construção do Índice de Qualidade de Vida Urbana dos municípios brasileiros (IQVU-BR). In: XV ENCONTRO NACIONAL DE ESTUDOS POPULACIONAIS, 2006, Caxambú. Anais... Caxambú: Associação de Estudos Populacionais (ABEP), 2006.

PARAHOS, Ranulfo; et al. Construindo indicadores sociais: uma revisão da bibliografia especializada. Perspectivas. 44: 147-173, jul./dez. 2013.

PAULA, Ana Paula Paes de. Por uma nova gestão pública: limites e potencialidades da experiência contemporânea. Rio de Janeiro: Editora FGV, 2005.

PRADO, Otávio; KEINERT, Tânia Margarete Mezzomo. Metodologia de implantação do Observatório da Qualidade de Vida de Santo André. In: VITTE, Claudete de Castro Silva; KEINERT, Tânia Margarete Mezzomo. Qualidade de vida, planejamento e gestão urbana: discussões teórico-metodológicas. Rio de Janeiro: Bertrand Brasil, 2009.

RODRIGUES, Arlete Moysés. 0 espaço urbano e as estratégias de planejamento e produção da cidade. In: PEREIRA, Elson Manoel. Planejamento urbano no Brasil: conceitos, diálogos e práticas. Chapecó: Argos, 2008.

SEN, Amartya. Desenvolvimento como liberdade. São Paulo: Companhia das Letras, 2010.

SOUZA, Amaury. Qualidade de vida urbana. Rio de Janeiro: Zahar, 1982.

VEIGA, José Eli da. Prefácio. In: VITTE, Claudete de Castro Silva; KEINERT, Tânia Margarete Mezzomo. Qualidade de vida, planejamento e gestão urbana: discussões teórico-metodológicas. Rio de Janeiro: Bertrand Brasil, 2009.

VITTE, Claudete de Castro Silva; et al. A experiência do projeto Observatório da Qualidade de Vida de Santo André (2000-2003). In: VITTE, Claudete de Castro Silva; KEINERT, Tânia Margarete Mezzomo. Qualidade de vida, planejamento e gestão urbana: discussões teórico-metodológicas. Rio de Janeiro: Bertrand Brasil, 2009.

VITTE, Claudete de Castro Silva; KEINERT, Tânia Margarete Mezzomo. Qualidade de vida, planejamento e gestão urbana: discussões teórico-metodológicas. Rio de Janeiro: Bertrand Brasil, 2009.

VITTE, Claudete de Castro Silva; et al. Novas abordagens de desenvolvimento e sua inserção na gestão de cidades. In: KEINERT, Tânia Margarete Mezzomo; KARRUZ, Ana Paula. Qualidade de vida: observatórios, experiências e metodologias. São Paulo: Annablume/FAPESP, 2002.

VITTE, Claudete de Castro Silva. A qualidade de vida urbana e sua dimensão subjetiva: uma contribuição ao debate sobre políticas públicas e a cidade. In: VITTE, Claudete de Castro Silva; KEINERT, Tânia Margarete Mezzomo. Qualidade de vida, planejamento e gestão urbana: discussões teórico-metodológicas. Rio de Janeiro: Bertrand Brasil, 2009. 